INPLASY

PROTOCOL

To cite: Yang et al. The efficacy of adding azithromycin to antibiotic prophylaxis in cesarean section: a metaanalysis and systematic review. Inplasy protocol 202150033. doi: 10.37766/inplasy2021.5.0033

Received: 09 May 2021

Published: 09 May 2021

Corresponding author: Shan Wang

shsh0811@163.com

Author Affiliation:

Jining First People's Hospital

Support: B-19-H-20200622.

Review Stage at time of this submission: Formal screening of search results against eligibility criteria.

Conflicts of interest:

None declared.

\section{The efficacy of adding azithromycin to antibiotic prophylaxis in cesarean section: a meta-analysis and systematic review}

Yang, M1; Yuan, F²; Guo, Y3; Wang, S4.

Review question / Objective: To explore the efficacy of adding azithromycin to antibiotic prophylaxis for patients undergoing CS, we conducted statistical analysis of related randomized controlled trials (RCTs) and cohort studies in the existing literature.

Condition being studied: Although prophylactic antibiotics are widely used, surgical site infections (SSI) of cesarean sections (CS) are still common. According to reports, the incidence of incision infection varies between $3 \%$ and $7 \%$, while the incidence of endometritis varies from $5 \%$ to $25 \%$. Studies have shown that SSI of CS may also be associated with mycoplasma. And some studies have shown that addition of azithromycin to antibiotic prophylaxis could reduce the risk of SSI in patients undergoing CS.

INPLASY registration number: This protocol was registered with the International Platform of Registered Systematic Review and Meta-Analysis Protocols (INPLASY) on 09 May 2021 and was last updated on 09 May 2021 (registration number INPLASY202150033).

\section{INTRODUCTION}

Review question / Objective: To explore the efficacy of adding azithromycin to antibiotic prophylaxis for patients undergoing CS, we conducted statistical analysis of related randomized controlled trials (RCTs) and cohort studies in the existing literature.

Condition being studied: Although prophylactic antibiotics are widely used, surgical site infections (SSI) of cesarean sections (CS) are still common. According to reports, the incidence of incision 
infection varies between $3 \%$ and $7 \%$, while the incidence of endometritis varies from $5 \%$ to $25 \%$. Studies have shown that SSI of CS may also be associated with mycoplasma. And some studies have shown that addition of azithromycin to antibiotic prophylaxis could reduce the risk of SSI in patients undergoing CS.

\section{METHODS}

Search strategy: In order to obtain relevant data, we will search various databases involving PubMed, Embase, the Cochrane Library, Web of Science, ClinicalTrials.gov, and Chinese databases (CNKI, WEIPU, WANFANG, China Biology Medicine disc [CBM], Chinese Clinical Trial Registry [ChiCTR]). We will identifiy relevant literature by using combinations terms, including "Cesarean Section", "Cesarean Sections", "Delivery, Abdominal" etc. and "Azithromycin", "Azythromycin", "Sumamed" etc. We will search databases for literature published between database inception and February 23, 2021. In addition, we also will check all identified articles carefully to see if we could capture additional relevant studies.

Participant or population: Patients who required cesarean section (including selective and non-selective cesarean section).

Intervention: Standard prophylactic antibiotic + azithromycin (any mode of administration).

Comparator: Standard prophylactic antibiotic \pm placebo (any mode of administration).

Study designs to be included: Cohort Studies and Randomized Controlled Trials.

Eligibility criteria: According to diagnostic criteria of Centers for Disease Control and Prevention.

Information sources: PubMed, Embase, the Cochrane Library, Web of Science, ClinicalTrials.gov, and Chinese databases (CNKI, WEIPU, WANFANG, China Biology
Medicine disc [CBM], Chinese Clinical Trial Registry [ChiCTR]).

Main outcome(s): Surgical site infections (including endometritis, wound infection and composite infections outcome).

Quality assessment / Risk of bias analysis: For randomized controlled trials : Two reviewers will independently assesses the quality of the selected studies according to the Cochrane Collaboration's tool. The following characteristics will be evaluated: random sequence generation (selection bias), allocation concealment (selection bias), blinding of participants and personnel (performance bias), blinding of outcome assessment (detection bias), incomplete outcome data (attrition bias), selective reporting (reporting bias), and other biases. For cohort studies: Two reviewers will independently assesses the quality of the selected studies according to the Newcastle-Ottawa Quality Scale (NOS). The following characteristics will be evaluated: selection, comparability, and outcome. Results from these questions will be graphed and assessed using Review Manager 5.3.

Strategy of data synthesis: Review Manager version 5.3 software was used for statistical analysis. The $Q$ test and the Chi squared test will be used to evaluate the heterogeneity between the original studies. $P<0.1$ or $12 \geq 50 \%$ will indicate that there is heterogeneity between the selected studies; in these cases, we will use a random effect model to merge the effect quantity, otherwise the fixed effect model will be used. The combined results of outcome indicators will be calculated by the combined statistical effect of relative risk (RR) and 95\% confidence interval (Cl). $P<0.05$ was considered to indicate statistical significance.

Subgroup analysis: We will consider subgroup such as population (selective and non-selective cesarean section). Since the data are dichotomous variables, it will be necessary to add the total number and the events number of each subgroup respectively when merging subgroup data. 
Sensitivity analysis: STATA 15.0 software was used for sensitivity analysis. The sensitivity of the data can be reflected by observing the change of effect quantity after excluding a certain data.

Language: English and Chinese.

Country(ies) involved: China.

Keywords: Azithromycin; Cesarean sections; Prophylactic antibiotics; Surgical site infections; Mycoplasma.

Contributions of each author:

Author 1 - Mengqi Yang.

Email: mengqiqiyang@163.com

Author 2 - Fang Yuan.

Email: 119075133@qq.com

Author 3 - Yujin Guo.

Email: guoyujin99@126.com

Author 4 - Shan Wang.

Email: shsh0811@163.com 